\title{
Free-body model for tolerance analysis of rigid parts with manufacturing signature and operating
} conditions

\author{
Wilma Polini $^{a^{*}}$ and Andrea Corrado ${ }^{a}$
}

${ }^{a}$ Università di Cassino e del Lazio Meridionale, Italy

\begin{tabular}{l}
\hline A R T I C L EI N F O \\
\hline Article history: \\
Received 7 July, 2019 \\
Accepted 6 August 2019 \\
Available online \\
6 August 2019 \\
\hline Keywords: \\
Free-body model \\
Manufacturing signature \\
Operating conditions \\
Tolerance analysis \\
Rigid bodies
\end{tabular}

\section{Introduction}

Engineering design widely uses tolerance analysis in order to verify the functionality, the manufacturability and the perceived quality of a product. Tolerance analysis estimates the resultant variation of the assembly geometry, due to the tolerances applied to the components and to the functional relationship between the components and the assembly requirements. It is needed to design and to manufacture a product. In fact, the standardization of production and the correct function of the assembly require an assignment of dimensional and geometric tolerances to assembly components. Lower costs per assembly and higher probability of fit, by reducing the number of rejected parts or the amount of rework required on components, are due to a right allocation of tolerances on the components of an assembly.

The literature presents many models for tolerance analysis. that are not completely and univocally accepted (Polini, 2012). Those tolerance analysis models for rigid assemblies are the variation model (Gupta \& Turner, 1993), the TTRS model (Desrochers, 2002), the matrix model (Desrochers \& Rivière, 1997), the vector loop (Gao et al., 1998), the jacobian model (Laperrière \& Lafond, 1999), the torsor model (Bourdet at al., 1996), the jacobian-torsor model (Ghie et al., 2003), the T-Map model (Davidson et al., 2002), the deviation domain model (Giordano et al., 1999). In the same time many commercial

* Corresponding author.

E-mail addresses: polini@unicas.it (W. Polini)

(C) 2019 Growing Science Ltd. All rights reserved. doi: $10.5267 /$ j.esm.2019.8.001 
software packages, that are based on some of the previously cited models, are available for tolerance analysis of rigid assemblies, such as MECAMaster ${ }^{\circledR}$, Sigmund ${ }^{\circledR}, 3 D C S^{\circledR}$, VisVSA ${ }^{\circledR}, \mathrm{CeTol}^{\circledR}$ and PolitoCAT $^{\circledR}$ (Prisco \& Giorleo, 2002). They efficiently deal with simple geometrical feature of mechanical assemblies, such as plane, hole-pin, while they hardly consider free-form surfaces to connect or form deviations. Skin Model Shape is a more recent model for tolerance analysis of rigid assemblies. It allows to deal with form deviation by discretizing the part surfaces by points (Schleich et al., 2014). A further recent tolerance analysis method uses the free-body diagrams of force analysis (Armillotta, 2014), thus translating a tolerance chain into a mechanical static problem, that is solved by common algebraic or graphical procedures. The proposed model, that takes advantage from its ease application with minimal software assistance, was applied to 2D tolerance analysis problems with only dimensional tolerances. Moreover, to bring the tolerance analysis methods near to reality, it is needed that they consider working conditions and operating windows (Armillotta \& Semeraro, 2013). Furthermore, a complete and coherent tolerancing process should incorporate all activities of geometric variations management (Charpentier et al., 2011). As a response to those needs the most important methods for tolerance analysis of rigid bodies were modified to take into account the manufacturing signature, i.e. the pattern left on the manufactured part surfaces by the adopted process, and the operating conditions during the assembly sequence (Corrado and Polini, 2017a, 2017b). This was possible thanks to the development of a Matlab ${ }^{\circledR}$ algorithm that, once generated, assembles bodies after evaluation of the contact stability between them. This algorithm was tested on a given case study to estimate the dimensional and form parameters in the actual contact points of the involved geometries. Once estimated these parameters, they were inserted in the equations of the considered rigid models.

The aim of this work was to propose a free body model that takes into account the manufacturing signature and the operating conditions during assembly. The new free body model deals with geometric tolerances together with dimensional ones. Finally, a sensitivity analysis was carried out to decrease the simulation time involved by the new free body model for tolerance analysis. The paper is organized as follow: in Section 2 the new free body model that deals with geometric and dimensional tolerances is presented. In section 3 the new free-body that deals with the manufacturing signature and the operating conditions involved by assembly is deeply described and tested. Finally, in section 4 an analysis of sensitivity is carried out.

\section{New free-body model with geometric tolerances}

The free-body model for tolerance analysis of rigid bodies uses the free-body diagrams of force analysis (Armillotta, 2015). It uses the sensitivity coefficients to evaluate the tolerance range of the functional requirement in the assembly by the tolerances applied to the single components. Those coefficients are sought from properties of equilibrium conditions on a rigid part, that reacts to an applied external force $F_{0}$ through the $N$ forces $F_{i}(i=1$ to $N)$ of the constrains. The contact points have small displacements $\delta_{i}$ along the directions of the reaction forces that involve a displacement $\delta$ of the body along the direction of the external force proportional to the reaction forces. This is similar to tolerance analysis, if the displacements $\delta_{i}$ are the manufacturing deviations and the resulting displacement $\delta$ is the stack-up of these deviations. The sensitivity coefficient of the resulting displacement to a deviation is evaluated through the equilibrium conditions of free-body diagrams developed for the assembly parts. Two unitary external forces with the same line of action and opposite orientations are associated on a couple of parts involved in a functional requirement.

This method was proposed to solve problems of tolerance analysis in which the parts were affected only by dimensional tolerances. The present work introduces a modified model able to consider geometric tolerances too under particular conditions. In fact, the proposed method translates the applied geometrical tolerances into translational variations, by taking into account the rotational and translational variations associated with the corresponding geometric tolerance-kinematic joint combinations (Polini \& Corrado, 2016), as explained in (Chase et al., 1996). 
A planar body is considered into contact with adjacent bodies in three frictionless points, that constrain its degrees of freedom; to its boundary an external force $\mathrm{F}$ is applied and the three constraints react with the forces $F_{1}, F_{2}$, and $F_{3}$. Once assumed rigid the body, by neglecting any force-induced deformations, the small displacements $\delta_{1}, \delta_{2}$, and $\delta_{3}$ of the contact points along the directions of the reaction forces involve a displacement $\delta$ of the point of force application, which is assumed to be positive if it is opposite to F. The body does not change its potential or kinetic energy, and, therefore, the total work of the four forces equals zero for the principle of virtual work of rigid bodies, yielding the following relationship among the four displacements:

$$
\delta=\frac{F_{1}}{F} \delta_{1}+\frac{F_{2}}{F} \delta_{2}+\frac{F_{3}}{F} \delta_{3} .
$$

Considering the rotational and translational variations due to the corresponding geometric feature tolerance-kinematic joint combinations, Eq. (1) becomes:

$$
\delta=\frac{F_{1}}{F}\left(\delta_{1}+\varepsilon_{1}\right)+\frac{F_{2}}{F}\left(\delta_{2}+\varepsilon_{2}\right)+\frac{F_{3}}{F}\left(\delta_{3}+\varepsilon_{3}\right),
$$

where $\varepsilon_{1}, \varepsilon_{2}, \varepsilon_{3}$ are the small displacements of the contact points due to the applied geometrical tolerances and $\delta_{1}, \delta_{2}$, and $\delta_{3}$ are the small displacements of the contact points due to the applied dimensional tolerances. $\varepsilon_{1}, \varepsilon_{2}, \varepsilon_{3}$ may be derived by the manufacturing signature, while $\delta_{1}, \delta_{2}$, and $\delta_{3}$ may be found by the applied dimensional tolerances.

\subsection{Application example}

In order to illustrate how the new free body model works, an assembly was used. The case study considers both dimensional and geometrical tolerances and, in the same time, it may be solved manually. This assembly was constituted by five components: a hollow rectangular box and four circular profiles inserted in it, as shown in Fig. 1. The aim is to measure the gap $g$ between the fourth circular profile and the top side of the box, once assigned the tolerances applied to the components.

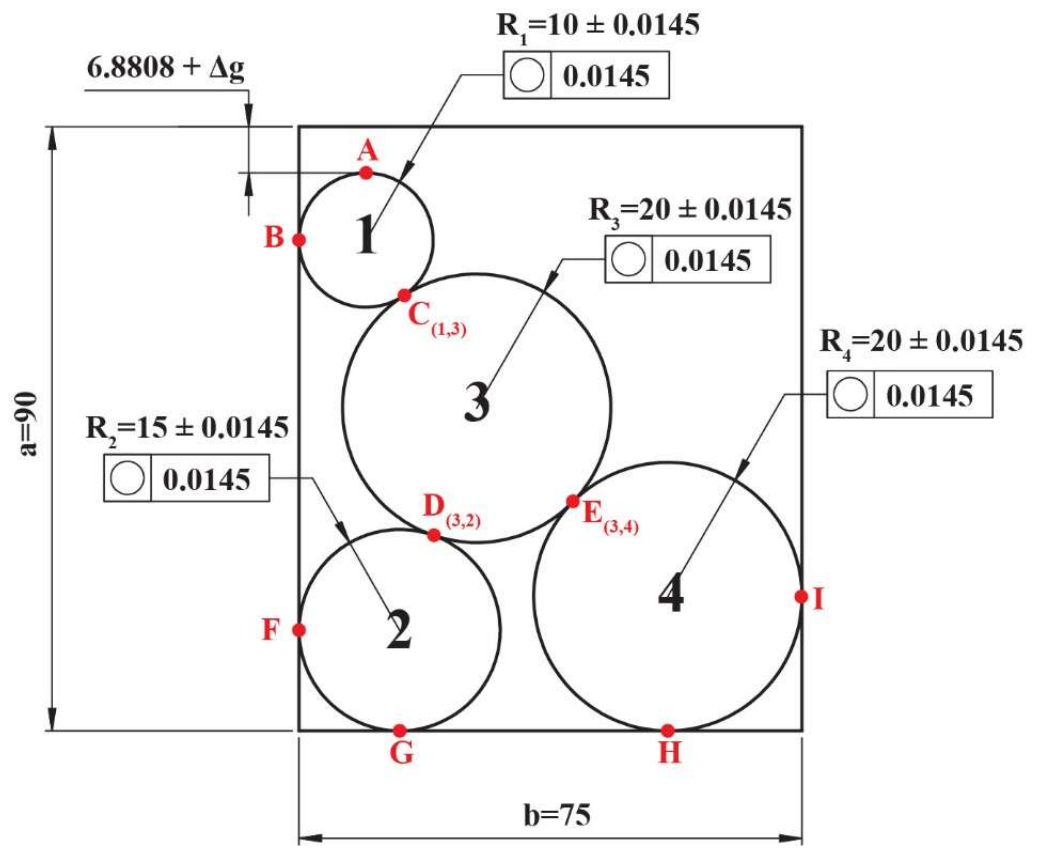

Fig. 1. Case study to be solved by free-body method (dimensions in $\mathrm{mm}$ ) 
The functional requirement $g$ depends on the dimensions $a$ and $b$ of the box, on the radius $R_{1}, R_{2}, R_{3}$, $R_{4}$ of the circular profiles and on the applied tolerances. The free-body diagram imposes the equilibrium conditions of the five parts and it obtains the decomposition of forces, as shown in Fig. 2. In this way it is possible to have the projection of the reaction forces along the directions of the functional dimensions. At this point, two kinds of sensitivities can be distinguished: a sensitivity due to the dimensional tolerances and a sensitivity due to the geometric tolerances. The sensitivity values of the dimensional tolerances are found through the following equation:

$$
S_{d}=\left[\begin{array}{c}
\partial g / \partial a \\
\partial g / \partial b \\
\partial g / \partial R_{1} \\
\partial g / \partial R_{2} \\
\partial g / \partial R_{3} \\
\partial g / \partial R_{4}
\end{array}\right]=\left[\begin{array}{c}
F_{0} \\
F_{5} \\
-F_{0}-F_{1}-F_{2} \\
-F_{4}-F_{7}-F_{8} \\
-F_{2}-F_{3}-F_{4} \\
-F_{3}-F_{5}-F_{6}
\end{array}\right]=\left[\begin{array}{c}
1.0000 \\
0.7494 \\
-2.8552 \\
-0.6314 \\
-2.5264 \\
-2.5385
\end{array}\right]
$$

The values of the sensitivities due to the geometric tolerances are evaluated in the points of the parts that affect the requirement, as shown in Fig. 1, and they are:

$$
S_{g}=\left[\begin{array}{l}
\partial g / \partial A \\
\partial g / \partial B \\
\partial g / \partial C_{1} \\
\partial g / \partial C_{3} \\
\partial g / \partial D_{3} \\
\partial g / \partial E_{3} \\
\partial g / \partial D_{2} \\
\partial g / \partial F \\
\partial g / \partial G \\
\partial g / \partial E_{4} \\
\partial g / \partial H \\
\partial g / \partial I
\end{array}\right]=\left[\begin{array}{l}
-F_{0} \\
-F_{1} \\
-F_{2} \\
-F_{2} \\
-F_{4} \\
-F_{3} \\
-F_{4} \\
-F_{8} \\
-F_{7} \\
-F_{3} \\
-F_{6} \\
-F_{5}
\end{array}\right]=\left[\begin{array}{l}
-1.0000 \\
-0.6581 \\
-1.1971 \\
-1.1971 \\
-0.2778 \\
-1.0515 \\
-0.2778 \\
-0.0912 \\
-0.2624 \\
-1.0515 \\
-0.7376 \\
-0.7494
\end{array}\right]
$$

because the geometric tolerances are considered as translational variations. The points $A, B$ and $C_{1}$ belong to the profile $1, C_{3}, D_{3}$ and $E_{3}$ belong to the profile $3, D_{2}, F$ and $G$ belong to the profile $2, E_{2}, H$ and $I$ belong to the profile 4 .
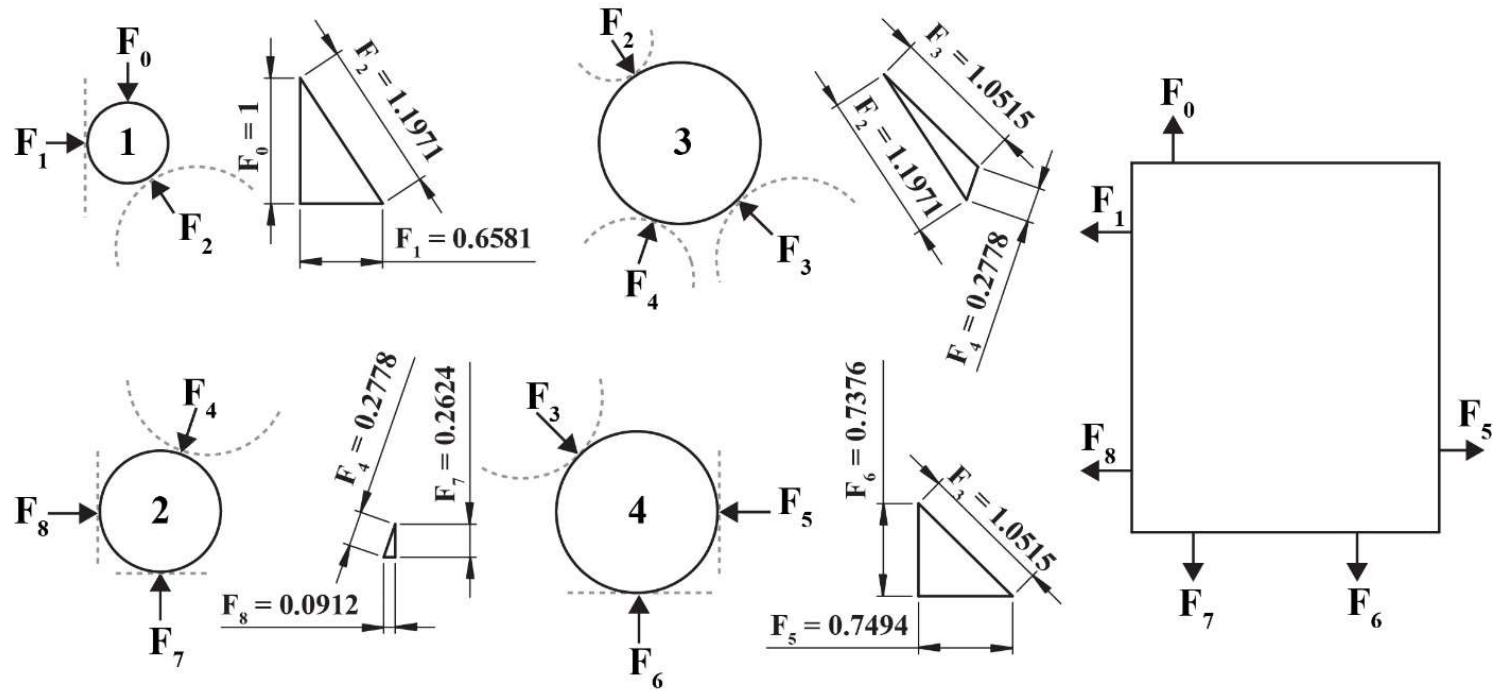

Fig. 2. Graphical calculations of the reaction forces 
The tolerance $\Delta g$ of the functional requirement $g$ may be evaluated from the tolerances assigned to parts and the sensitivity coefficients $S_{i}$ by using the worst-case or the statistical (root sum square - RSS) stack-up:

$$
\begin{aligned}
& \text { Worst Case : } \Delta g=\sum_{i=1}^{n}\left|S_{i}\right|^{T} \cdot T_{i} \\
& \text { RSS : } \Delta g=\sqrt{\sum_{i=1}^{n}\left(\left(S_{i}\right)^{T}\right)^{2} \cdot\left(T_{i}\right)^{2}}
\end{aligned}
$$

where $n$ is the number of considered tolerances, $S$ and $T$ are the total sensitivities and all dimensional and geometrical tolerances of parts respectively. In particular, $S$ and $T$ are equal to:

$$
\begin{gathered}
S=\left[\begin{array}{l}
S_{d} \\
S_{g}
\end{array}\right] \\
T=\left[\begin{array}{l}
T_{d} \\
T_{g}
\end{array}\right]
\end{gathered}
$$

with $T_{d}$ and $T_{g}$ that are considered as following:

$$
\begin{aligned}
& T_{d}=\left[\begin{array}{llllll}
0 & 0 & r_{1} & r_{2} & r_{3} & r_{4}
\end{array}\right]^{T} \\
& T_{g}=\left[\begin{array}{llllllllllll}
d_{A} & d_{B} & d_{C_{1}} & d_{C_{3}} & d_{D_{3}} & d_{E_{3}} & d_{D_{2}} & d_{F} & d_{G} & d_{E_{4}} & d_{H} & d_{I}
\end{array}\right]^{T}
\end{aligned}
$$

where $r_{1}, r_{2}, r_{3}$ and $r_{4}$ are the parameters connected to the dimensional tolerance $( \pm 0.0145)$ of the four circular profiles, $d_{i}$ is the form tolerance $( \pm 0.0145 / 2)$ parameter applied to the points $i=A, B$ and $C_{1}$ of the profile $1, C_{3}, D_{3}$ and $E_{3}$ of the profile $3, D_{2}, F$ and $G$ of the profile 2, $E_{2}, H$ and $I$ of the profile 4. So, solving Eq. (3), $\Delta g$ is equal to:

$$
\begin{aligned}
& \text { Worst Case }: \Delta g=\sum_{i=1}^{n}\left|S_{i}\right|^{T} \cdot T_{i}= \pm 0.1860 \mathrm{~mm} \\
& \text { RSS : } \Delta g=\sqrt{\sum_{i=1}^{n}\left(\left(S_{i}\right)^{T}\right)^{2} \cdot\left(T_{i}\right)^{2}}= \pm 0.0701 \mathrm{~mm}
\end{aligned}
$$

Moreover, a Monte Carlo simulation may be used to estimate the functional requirement and its variation. In this case, the stack-up function is:

$$
g=6.8808+(S)^{T} \cdot T
$$

and the parameters connected with the dimensional $\left(r_{1}, r_{2}, r_{3}\right.$ and $\left.r_{4}\right)$ or the form $\left(d_{i}\right)$ tolerances have a Gaussian probability density function with a mean value equal to zero and a standard deviation equal to a sixth of the tolerance range. 50,000 simulation runs were carried out and the obtained functional requirement was equal to $6.8807 \pm 0.0702 \mathrm{~mm}$. The same tolerance chain solved by dealing with only dimensional tolerances gives:

$$
\begin{aligned}
& \text { Worst Case : } \Delta g=\sum_{i=1}^{n}\left|S_{i}\right|^{T} \cdot T_{i}= \pm 0.1240 \mathrm{~mm} \\
& \text { RSS : } \Delta g=\sqrt{\sum_{i=1}^{n}\left(\left(S_{i}\right)^{T}\right)^{2} \cdot\left(T_{i}\right)^{2}}= \pm 0.0670 \mathrm{~mm}
\end{aligned}
$$

Therefore, the results due to the geometrical tolerances involve an increase of $\Delta g$ of about $50 \%$ and $5 \%$ regards to the results due to the dimensional tolerances, if worst-case and statistical methods are applied respectively. 


\section{New free body method with geometrical tolerances, manufacturing signature and operation conditions}

\subsection{Manufacturing signature and operating conditions}

The new free body model was modified to insert the manufacturing signature and the operating condition. Moreover, since an analysis on the number of points used to discretize the bodies was carried out too, a subpart of the previous assembly was used (see Fig. 3a) to better explain the implementation by preserving the generality of the approach. This sub-assembly was constituted by three components: a hollow rectangular box into which two circular profiles are inserted. The aim was the measurement of the gap $g$ between the top side of the box and the second circular profile even in this case.

For this sub-assembly, the decomposition of the forces carried out by the free body diagram (see Fig. $3 b$ ) allows to determine the following sensitivity coefficients connected with dimensional and geometric tolerances:

$$
\begin{aligned}
S_{d}= & {\left[\begin{array}{c}
\partial g / \partial a \\
\partial g / \partial b \\
\partial g / \partial R_{1} \\
\partial g / \partial R_{2}
\end{array}\right]=\left[\begin{array}{c}
F_{0} \\
F_{1} \\
-F_{0}-F_{1}-F_{2} \\
-F_{3}-F_{4}-F_{2}
\end{array}\right]=\left[\begin{array}{c}
1 \\
0.2582 \\
-2.2910 \\
-2.2910
\end{array}\right] } \\
S_{g}= & {\left[\begin{array}{l}
\partial g / \partial A \\
\partial g / \partial B \\
\partial g / \partial C_{1} \\
\partial g / \partial C_{2} \\
\partial g / \partial D \\
\partial g / \partial E
\end{array}\right]=\left[\begin{array}{l}
-F_{4} \\
-F_{3} \\
-F_{2} \\
-F_{2} \\
-F_{1} \\
-F_{0}
\end{array}\right]=\left[\begin{array}{c}
-1 \\
-0.2582 \\
-1.0328 \\
-1.0328 \\
-0.2582 \\
-1
\end{array}\right] }
\end{aligned}
$$

and the stack-up function:

$$
g=1.2702+(S)^{T} \cdot T
$$

The results of the sub-assembly are:

Worst Case : $\Delta g= \pm 0.0997 \mathrm{~mm}$

RSS : $\Delta g= \pm 0.0493 \mathrm{~mm}$

Monte Carlo: $\Delta g= \pm 0.0492 \mathrm{~mm}$ considering 50,000 runs

considering as $T_{d}$ and $T_{g}$ the following relation:

$$
\begin{aligned}
T_{d} & =\left[\begin{array}{llll}
0 & 0 & r_{1} & r_{2}
\end{array}\right]^{T} \\
T_{g} & =\left[\begin{array}{llllll}
d_{A} & d_{B} & d_{C_{1}} & d_{C_{2}} & d_{D} & d_{E}
\end{array}\right]^{T}
\end{aligned}
$$

where $r_{1}$ and $r_{2}$ are the parameters of the dimensional tolerance $( \pm 0.0145)$ assigned to the two circular profiles, $d_{i}$ is the parameter of the form tolerance $( \pm 0.0145 / 2)$ applied to the points $i=A, B$ and $C_{1}$ of the profile $1, C_{2}, D$ and $E$ of the profile 2 . 

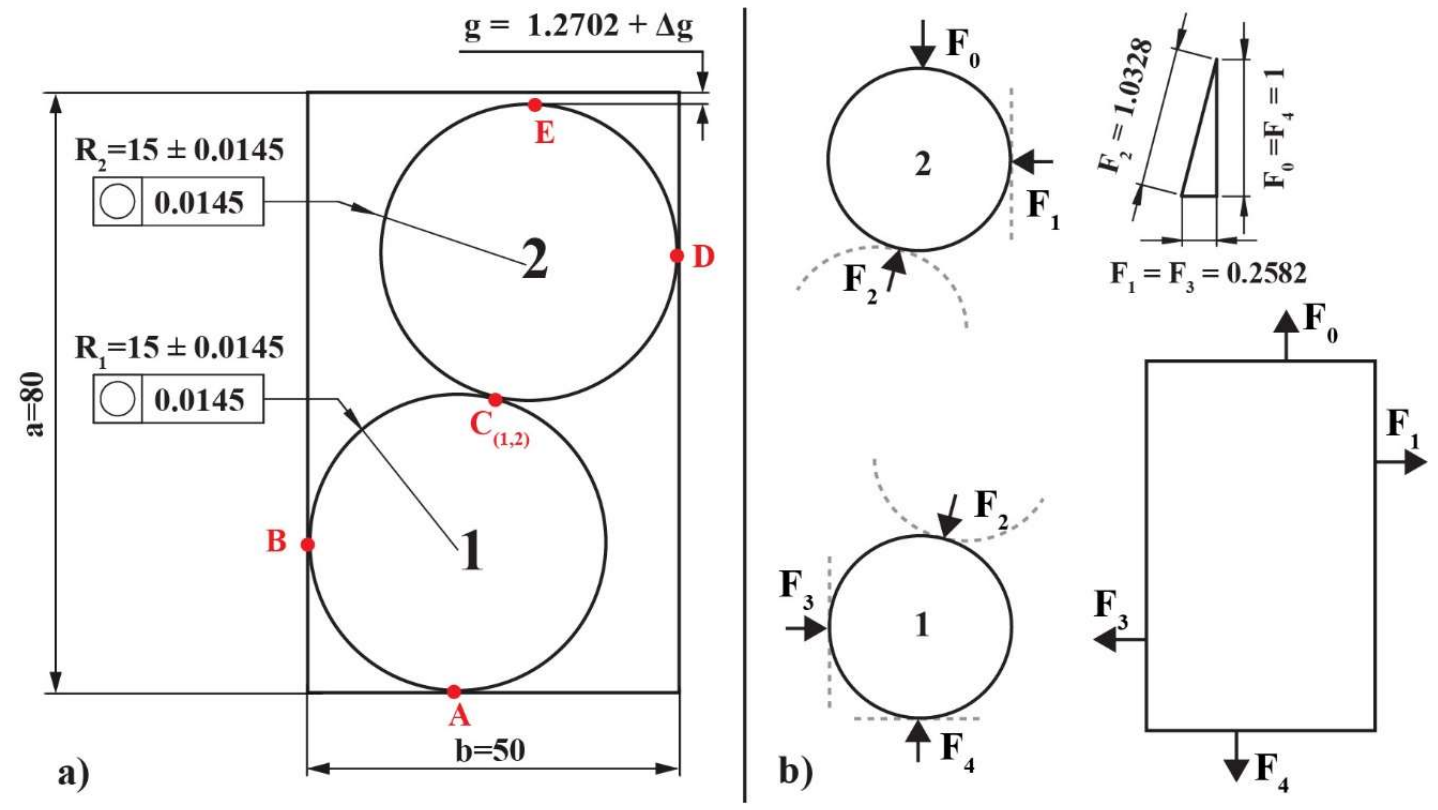

Fig. 3. - a) Sub-assembly of the case study (dimensions in $\mathrm{mm}$ ), b) Graphical calculations of the reaction forces.

The first step was the generation of each circular profile to put into the hollow rectangular box and their casual rotation. The circular profiles were discretized by 7,150 evenly distributed points. For each point of the circular profile it is valid the following model:

$$
\left|P_{i}-O\right|=R+r+d
$$

where $P_{i}$ was the circular profile generic point, $O$ was the circle centre, $R$ was the nominal value of the circular profile radius, $r$ was the dimensional tolerance parameter of the circular profiles, and $d$ was the ARMAX model parameter representing the manufacturing signature. In fact, the manufacturing signature affected mainly by both bi-lobe and three-lobe contours was represented by an autoregressive (AR) moving-average (MA) model with exogenous (X) variable (Moroni and Pacella, 2008). It is constituted by an harmonic term representing the systematic pattern left by the turning process on the manufactured surface and the a random contribution that may not be expected by a second-order autoregressive of the noise term. Therefore, the manufacturing signature represents the values of the form tolerance of the four circular profiles. These obtained values were used to define the values of the parameters in the vector $T_{g}$. The parameters in the vector $T_{d}$, due to the dimensional tolerance, were simulated as before.

The coordinates of the centres of gravity were also evaluated. In fact, being known the $\mathrm{x}$ and $\mathrm{y}$ coordinates of the 7,150 points of a generic profile, it was possible to determine the coordinates of the centre of gravity. The profiles were discretized as the set of $(7,150-1)$ triangles whose vertex coordinates: $\mathrm{v}_{1}, \mathrm{v}_{2}$, and $[0,0]$ were known. Note the position of the vertices, the side lengths $\mathrm{a}, \mathrm{b}$ and $\mathrm{c}$ of the triangles were determined (Fig. 4). At this point, it was possible to evaluate the centres of gravity $(G)$, the semiperimeters, the areas (by Heron's Formula) and the static moments of inertia of the triangles. With this information, the area and the static moment of the generic profile were calculated as the sum of the areas and the static moments of the triangles and so it was possible to evaluate the centre of gravity of the generic profile.

Once assembled, to evaluate if the general position of each circular profile was stable, the model evaluates the balance condition between the forces by requiring that they pass through the same point. Therefore, the weight force was applied in the centre of gravity of the profiles (barycentres $G_{1}$ and $G_{2}$ in Fig 5), the reactions were applied to the points of contact between the contours, and they were directed 
toward the centre of gravity of the relevant profile. $\beta_{1}, \beta_{2}, \beta_{3}, \beta_{4}$ and $\beta_{5}$ were the angles among these reactions and the normal vectors to the surfaces as shown in Fig. 5, whose values should be smaller than the static friction limit angle in order to have a stable position of the circular profiles. Otherwise, the circular profile rotated until the values became smaller. Once verified the two circular profiles were stable, the coordinates of the actual contact points among the four circular profiles and the box were obtained by considering the dimensional and form deviations. The values of the parameters $d_{i}, r_{1}$ and $r_{2}$ were substituted into Eq. (11) in order to have the value of the $g$ gap. Also in this case, 50,000 Monte Carlo simulation runs were carried out and the obtained functional requirement was equal to $1.2702 \pm$ $0.0535 \mathrm{~mm}$.

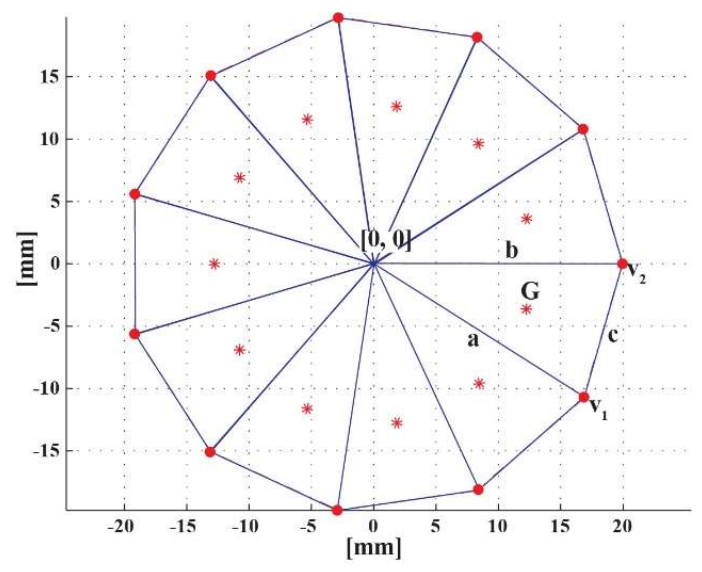

Fig. 4. Evaluation of the centre of gravity

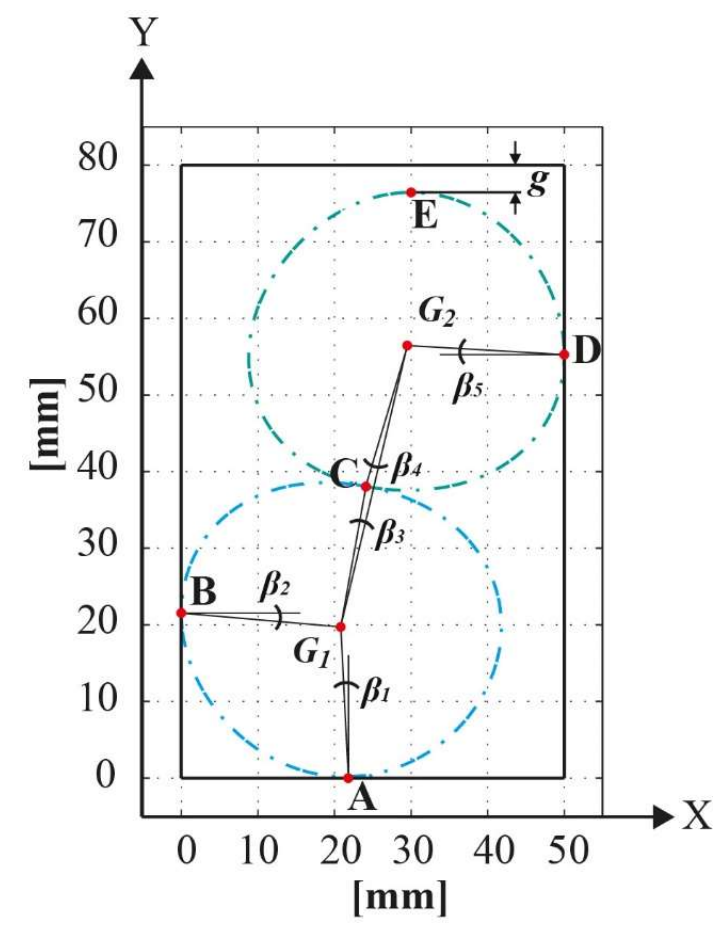

Fig. 5. $\beta$ angles to evaluate if each circular profile is stable

\section{Sensitivity analysis}

The simulation time is the main limit of the free body model with geometric tolerances, manufacturing signature and operating conditions. In fact, as previously shown in (Corrado et al., 2017), the free body model without manufacturing signature and operating conditions, employs very few seconds to make 50,000 simulation runs. In contrast, the free body model with manufacturing signature and operating conditions employs about 3 hours to carry out the same number of simulation runs. For this reason, a first attempt to reduce the simulation time consisted in calculating the centres of gravity of the profiles in a different way. In fact, by calculating the coordinates of the centres of gravity with the following equation:

$$
\begin{aligned}
& x_{G_{j}}=\sum x_{i} / N_{\text {points }} \\
& y_{G_{j}}=\sum y_{i} / N_{\text {points }}
\end{aligned}
$$

where $x_{G j}$ and $y_{G j}$ are the centre of gravity coordinates of a generic profile $j, N_{\text {points }}$ are the number of points that constitute the profile (7,150 in this case), $x_{i}$ and $y_{i}$ are the coordinates of the profile points (with $i=1$ to $\left.N_{\text {points }}\right)$ and, repeating the simulations with the same number of discretization points $(7,150$ for each profile) and simulation runs $(50,000)$, the simulation time decreases, as shown in Fig. 6, if compared to the reference model (Reference). Moreover, this change does not alter the outcome of the 
functional requirement ( $g$ gap) and the angular values $(\beta)$ in the points of contact, as shown in Figs. 7 and 8.

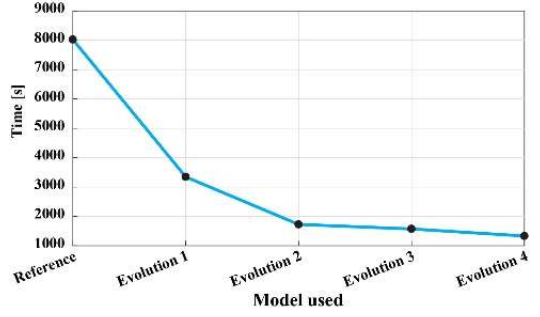

Fig. 6. Simulation times

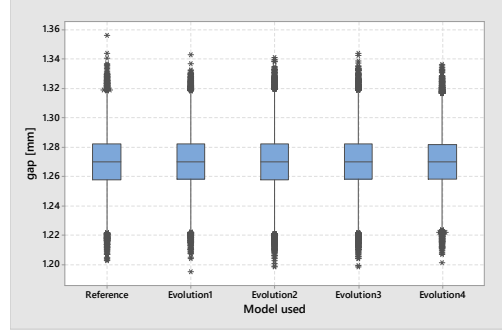

Fig. 7. Results of the functional requirement $g$ for all used models

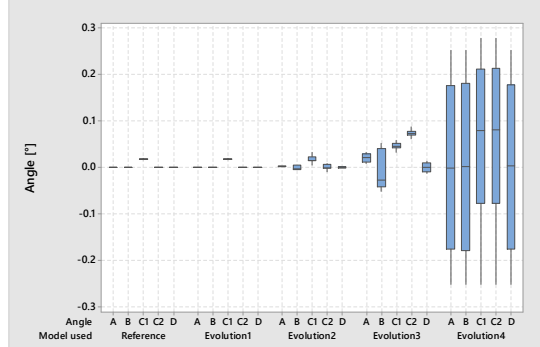

Fig. 8. Results of the angles $\beta$ for all used models

Then, simulations considering 7,150 (Evolution 1), 748 (Evolution 2) points and 80 (Evolution 3) points to discretize each circular profile were carried out, repeating 50,000 runs. Analysing the results of the functional requirement ( $g$ gap) in all simulations that are carried out, there are no significant variations. Instead, the angular values $(\beta)$ in the contact points tend to increase, when the points used to discretize the profiles decrease, as shown in Fig. 7 and Fig. 8. Moreover, the simulation times decrease with the number of points, as shown in Fig. 6. This aspect happens due to the reduced number of points used to discretize the profiles that determine a less accurate evaluation of the centre of gravity and, then, the angles in the contact points. In particular, the use of 748 discretization points allows to reduce the simulation time and to have very similar results to the simulations carried out considering 7,150 points, in term of the functional requirement and angles.

Another step is to consider only the points of contact among the components on the circular profiles (Evolution 4). This means that the profile 1 is exclusively represented by points $A, B$ and $C$, and the profile 2 is exclusively represented by the points $C, D$ and $E$. The random rotations to assign to each profile and the evaluation of the stability cannot take into count if only three points are considered on each profile. To overcome this drawback, the model was modified even further. In fact, in this case, the three points on each profile are located in their nominal position and the nominal centre of gravity of each profile was evaluated by means of Eq. (15). Subsequently, the points on each profile are rotated randomly and in this position, the form errors, to be included in Eq. (11), and the coordinates of the centre of gravity in the actual position are obtained. The angles between the nominal and real centres of gravity and the nominal coordinates of the points represent the friction angles for the evaluation of stability.

Repeating 50,000 runs of simulation, the simulation time decreases, as shown in Fig. 6. Considering all simulation times, an asymptotic trend is highlighted, then an inferior limit in the computation cost of the involved operations in the Matlab $^{\circledR}$ algorithm rises. Analysing the result of the functional requirement ( $g$ gap), there is no significant variations compared to other model, as shown in Fig. 7. Instead, the angular values $(\beta)$ in the contact points increase significantly both in terms of mean and standard deviation, as shown in Fig. 8. However, the ranges of variation of the angular values in the contact points is very small, $0.4^{\circ}$, and significantly smaller than the friction angle used in this work.

A sensitivity analysis was carried out by varying the number of simulation runs, such as shown in Fig. 9. The mean value and the standard deviation of all the obtained results are stable for 50.000 runs, that is the value considered in all the investigations of this paper. 


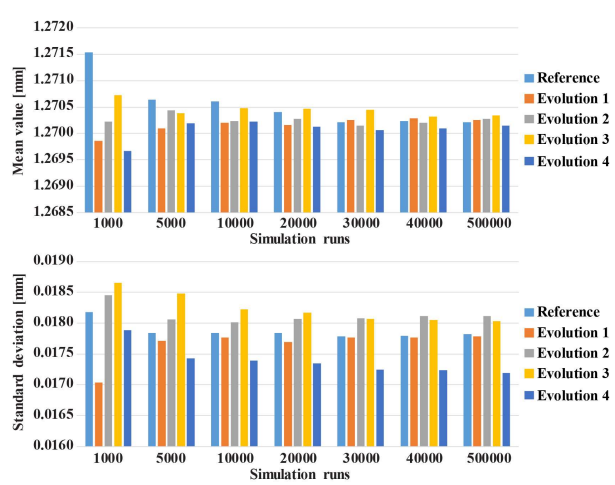

Fig. 9. Results vs simulation runs

\subsection{Sensitivity analysis results applied to the case study}

The sensitivity analysis shows that by using only the points of contacts for each profile, the simulation times are reduced without losing accuracy on results. For this reason, the case study was solved with this approach. Eq. (7) was also solved by considering the manufacturing signature, i.e. the form tolerance values of four circular profiles were substituted by the manufacturing signature values due to the ARMAX model. These obtained values were used to define the values of the parameters in the vector $T_{g}$. The parameters in the vector $T_{d}$, due to the dimensional tolerance, were simulated as before. Because by 3 points are considered on each profile, but one of these is in contact with no body (point $A$ shown in Fig. 1 ), the $\beta$ angles to be controlled are 11 and they are indicated on the basis of the designation of the contact point (for example, $\beta_{C}, \beta_{D}$, etc). Also in this case, 50,000 Monte Carlo simulation runs were carried out and the obtained functional requirement was equal to $6.8808 \pm 0.0727 \mathrm{~mm}$. The free-body model gives a value of the standard deviation that is lower of about $4 \%$ than that due to free-body model with manufacturing signature (Free-body modified), since this model considers nominal contact points among the components during assembling and it does not take into account the manufacturing signature. This conclusion is verified by the Levene test too (see Fig. 10) and it is in agreement with what was said in previous works. A first indication on the possible stability of the bodies involved in the assembling is given by the angular values $(\beta)$ in the contact points, as shown in Fig. 11.

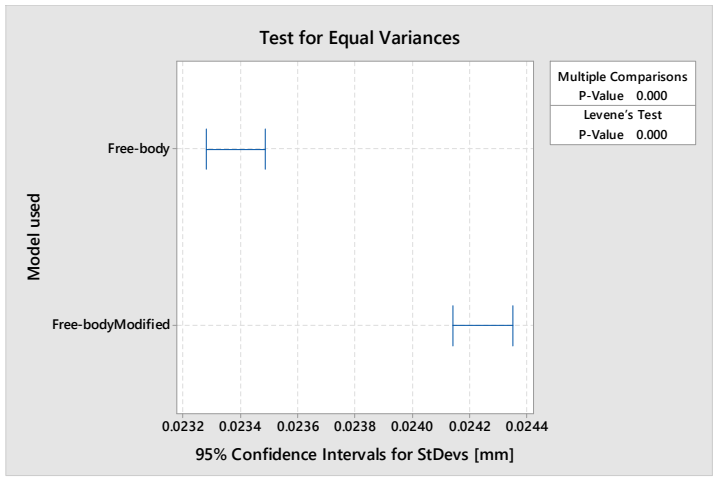

Fig. 10. Tests for equal variances

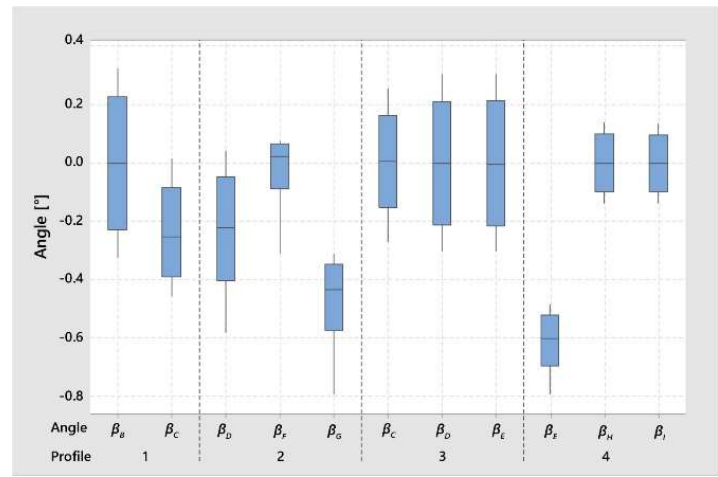

Fig. 11. Results of the angles $\beta$ for the modified free-body method

\section{Conclusions}

This paper proposed a free-body model that simulate the geometrical tolerances applied to the assembly components under particular conditions. In fact, it translates the applied geometrical tolerances in translational variations, by considering the rotational and translational variations associated with 
corresponding geometric feature tolerance-kinematic joint combinations. Moreover, the new free body model simulates the manufacturing signature and the operating conditions during the tolerance analysis too. A sensitivity analysis on the new free body model demonstrated that the model that considers only the points of contact for tolerance analysis with manufacturing signature and operating conditions gives good performances in terms of simulation time and reliability in the evaluation of the stability of the contacts among the parts. The results show that considering manufacturing signature and operating conditions inside the free-body model allows to improve the digital twin of the actual machined circular profiles assembling; in fact, the standard deviation of the functional requirement is greater of about $4 \%$ than that without manufacturing signature. Future works aim to make the free-body model able to simulate all geometric tolerances together with the dimensional ones without the considered constrains taken into account in the present work.

\section{References}

Ameta, G., Serge, S., \& Giordano, M. (2011). Comparison of spatial math models for tolerance analysis: tolerance-maps, deviation domain, and TTRS. Journal of Computing and Information Science in Engineering, 11(2), 021004.

Armillotta, A., \& Semeraro, Q. (2013). Critical operating conditions for assemblies with parameterdependent dimensions. Proceedings of the Institution of Mechanical Engineers, Part B: Journal of Engineering Manufacture, 227(5), 735-744.

Armillotta, A. (2014). A static analogy for 2D tolerance analysis. Assembly Automation, 34(2), 182-191.

Armillotta, A. (2015). Force analysis as a support to computer-aided tolerancing of planar linkages. Mechanism and Machine Theory, 93, 11-25.

Bourdet, P., Mathieu, L., Lartigue, C., \& Ballu, A. (1996). The Concept of the Small Displacement Torsor in Metrology. Series on Advances in Mathematics for Applied Sciences, 40, 110-122.

Charpentier, F., Ballu, A., \& Pailhes, J. (2011). A scientific point of view of a simple industrial tolerancing process. Procedia Engineering, 1, 1-10.

Chase, K.W., Gao, J., Magleby, S.P., \& Sorensen, C.D. (1996). Including geometric feature variations in tolerance analysis of mechanical assemblies. IIE Transactions, 28, 795-807.

Corrado, A., \& Polini, W. (2017). Manufacturing signature in variational and vector-loop models for tolerance analysis of rigid parts. International Journal of Advanced Manufacturing Technology, 88(58), 2153-2161.

Corrado, A., Polini, W., \& Moroni, G. (2017). Manufacturing signature and operating conditions in a variational model for tolerance analysis of rigid assemblies. Research in Engineering Design, 28(4), 529-544.

Corrado, A., \& Polini, W. (2017). Manufacturing signature in jacobian and torsor models for tolerance analysis of rigid parts. Robotics and Computer-Integrated Manufacturing, 46, 15-24.

Davidson, J.K., Mujezinović, A., \& Shah, J.J. (2002). A New Mathematical Model for Geometric Tolerances as Applied to Round Faces. Journal of Mechanical Design, 124(4), 1-10.

Desrochers, A., \& Rivière, A.A. (1997). Matrix approach to the representation of tolerance zones and clearances. International Journal of Advanced Manufacturing Technology, 13(9), 630-36.

Desrochers, A.A. (2002). CAD/CAM representation model applied to tolerance transfer methods. Journal of Mechanical Design, 125(1), 1-10.

Gao, J., Chase, K.W., \& Magleby, S.P. (1998). Generalized 3-D tolerance analysis of mechanical assemblies with small Kinematic adjustments. IIE Transactions, 30(4), 367-77.

Ghie, W., Laperrière, L., \& Desrochers, A.A. (2003). Unified Jacobian-Torsor Model for Analysis in Computer Aided Tolerancing. In G. Gogu, D. Coutellier, P. Chedmail, \& P. Ray (eds.), Recent Advances in Integrated Design and Manufacturing in Mechanical Engineering (p. 63-72). Dordrecht: Springer Netherlands.

Giordano, M., Pairel, E., \& Samper, S. (1999). Mathematical representation of tolerance zones. In F. van Houten, \& H. Kals (Eds.), Global Consistency of Tolerances: Proceedings of the 6 Th CIRP International Seminar on Computer-Aided Tolerancing, University of Twente, Enschede, The 
Netherlands, 22--24 March, 1999 (p. 177-186). Dordrecht: Springer Netherlands.

Gupta, S., \& Turner, JU. (1993). Variational Solid Modeling for Tolerance Analysis. IEEE Computer Graphics and Applications, 13(3), 64-74.

Laperrière, L., \& Lafond, P. (1999). Tolerance Analysis And Synthesis Using Virtual Joints. In F. van Houten, \& H. Hubert Kals (Eds.), Global Consistency of Tolerances (p. 405-414). Dordrecht: Springer Netherlands, 1999, pp. 405-14.

Moroni, G., \& Pacella, M. (2008). An approach based on process signature modeling for roundness evaluation of manufactured items. Journal of Computing and Information Science in Engineering, 8(2), 021003.

Polini, W. (2012). Taxonomy of models for tolerance analysis in assembling. International Journal of Production Research, 50(7), 2014-2029.

Polini, W., \& Corrado, A. (2016). Geometric tolerance analysis through Jacobian model for rigid assemblies with translational deviations. Assembly Automation, 36(1), 72-79.

Prisco, U., \& Giorleo, G. (2002). Overview of current CAT systems. Integrated Computer-Aided Engineering, 9(4), 373-387.

Schleich, B., Anwer, N., Mathieu, L., \& Wartzack, S. (2014). Skin model shapes: A new paradigm shift for geometric variations modelling in mechanical engineering. Computer-Aided Design, 50, 1-15.

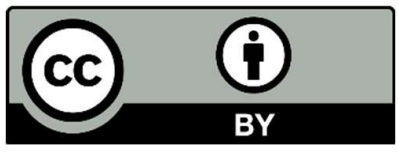

(C) 2019 by the authors; licensee Growing Science, Canada. This is an open access article distributed under the terms and conditions of the Creative Commons Attribution (CC-BY) license (http://creativecommons.org/licenses/by/4.0/). 